\title{
Assessments of Fusarium Head Blight of Wheat and Barley in Response to Fungicide Treatment
}

\author{
R. K. Jones, Professor and Extension Plant Pathologist, Department of Plant Pathology, University of Minnesota, \\ St. Paul 55108
}

\begin{abstract}
Jones, R. K. 2000. Assessments of Fusarium head blight of wheat and barley in response to fungicide treatment. Plant Dis. 84:1021-1030.

Benomyl and tebuconazole reduced head blight incidence, head blight severity, and the percentage of visually scabby kernels (VSK) in harvested grain when applied to the susceptible spring wheat cv. Norm at anthesis. Deoxynivalenol (DON) concentration was also reduced. Fungicide treatment increased head weight, test weight, thousand kernel weight, and yield. Fludioxonil reduced certain parameters associated with the disease in barley, including incidence, severity, and deoxynivalenol concentration, while increasing the percentage of plump kernels and yield. In vitro, isolates of Fusarium graminearum were sensitive to fludioxonil, benomyl, tebuconazole, and mancozeb (concentrations that give 50\% growth inhibition of $7.4 \times 10^{-3}, 3.5 \times 10^{-1}$, $9.9 \times 10^{-1}$, and $7.3 \mathrm{mg}$ a.i./liter, respectively). No evidence for insensitivity (resistance) was found. Prospects for chemical control of Fusarium head blight (FHB), however, remain limited. The repeatability of assessment methods used in evaluating host response to fungicides and to the damage caused by $F$. graminearum is discussed. Disease incidence, disease severity, VSK, and DON were identified as key variables that best measure the effects of FHB.
\end{abstract}

Additional keywords: chemical control, Fusarium graminearum, Gibberella zeae

Fusarium head blight (FHB) or scab has reemerged as one of the most significant problems affecting wheat (Triticum aestivum L.) and barley (Hordeum vulgare L.) production in the Upper Midwest (21). In Minnesota, FHB is caused primarily by Fusarium graminearum Schwabe Group 2 (teleomorph Gibberella zeae (Schwein.) Petch; 37,39). Disease loss results from direct reductions in yield and test weight, and by price discounting due to the presence of Fusarium spp.-damaged kernels and the associated mycotoxin, deoxynivalenol (DON; 18,21). Efforts to manage losses through conventional methods, including crop rotation and thorough tillage, have not proven entirely successful (9). Rotation options are limited in cereal-intensive production areas that cultivate substantial proportions of host crops. Also, complete tillage (moldboard plowing in the fall followed by disking operations in the fall or spring) is incompatible with recently adopted minimum-till and reduced-till

Corresponding author: R. K. Jones

E-mail: rogerj@puccini.crl.umn.edu

Published as paper $991,220,090$ of the contribution series of the Minnesota Agricultural Experiment Station, based on research conducted under project 22-038.

Accepted for publication 8 June 2000 .

Publication no. D-2000-0717-02R

(C) 2000 The American Phytopathological Society systems (1). Drier weather and fewer plantings of susceptible spring wheat cultivars have reduced losses in recent years compared with epidemics experienced during the early 1990s (18), but the number of moderately resistant (MR) cultivars is very limited $(15,34,36)$ and most moderately susceptible (MS) cultivars exhibit susceptibility to Septoria diseases. There are no moderately resistant or resistant cultivars of 6-row malting barley currently available and approved by the American Malting Barley Association. Although cultivar development continues in regional spring wheat and malting barley programs, additional control options are needed.

The efficacy of fungicides to control FHB or to reduce damage caused by Fusarium spp. in small grains has been examined in several published reports dating back to 1977 (14). Reductions in FHB of up to $66 \%$ have been reported (32), although limited or marginal response to treatment is more typically observed $(19,23,35)$. Jacobsen (14) reported a reduction in the number of Fusarium spp.infected kernels from 24.3 to $6.8 \%$ in plots of soft red winter wheat treated with high rates $(0.55$ to $1.10 \mathrm{~kg} / \mathrm{ha})$ of benomyl or carbendazim at heading. Boyacioglu et al. (7) reported an $83 \%$ reduction in DON contamination of spring wheat sprayed with thiabendazole at $360 \mathrm{~g}$ a.i./ha 2 days prior to inoculation. However, weather conditions in this study were very unfavorable for disease development, and symptoms of FHB were not observed in the test plots (7). The reduction in DON represented a change of $1.63 \mu \mathrm{g} / \mathrm{g}$ (control) to $0.27 \mu \mathrm{g} / \mathrm{g}$ (thiabendazole treated) but was not accompanied by a reduction in the number of Fusarium spp.-infected kernels (7). Difficulty exists in evaluating the sparse literature involving chemical control of FHB because disease levels are extremely variable between studies, and treatment effects are often reported on the basis of dissimilar parameters that are inconsistently measured (35).

Effective and consistent chemical control of FHB may require application of fungicides directly to headed wheat and barley $(21,35)$. Such applications raise serious concerns about residues of these compounds in food, beverage, and feed products, particularly because some of these fungicides are suspected or potential oncogens (3). Weighed against these factors are the fate and potential detriment posed to consumers by mycotoxins, including DON, which are present to varying levels in Fusarium spp.-damaged grain $(8,18,21)$. An integrated approach to managing FHB that includes chemical, cultural, and host-resistance factors seems the most logical way to reduce catastrophic losses that result from disease outbreaks. Crop rotation practices can and are being altered in Minnesota and elsewhere; however, recommendations involving the use of complete tillage are contentious in light of recent soil conservation mandates $(1,9)$. Further, the inheritance and manipulation of host resistance is complex and only moderate gains have been realized $(34,36)$. Effective chemical control options may become necessary to supplement available management options, particularly during growing seasons when environmental conditions are highly favorable for FHB.

In a 1993 demonstration trial, 18\% fewer Fusarium spp.-damaged heads and 9.4\% fewer Fusarium spp.-damaged kernels were observed in three hard red spring wheat (HRSW) cultivars sprayed with mancozeb (Dithane 75DF at $1.68 \mathrm{~kg}$ a.i./ha) at heading (Feekes growth stage 10.5 ; 29) compared with treatments sprayed prior to that time (Feekes stages 5.0 or 8.0) or those receiving no fungicide (R. K. Jones, unpublished data). Treatments receiving heading applications produced greater yields (by $411 \mathrm{~kg} / \mathrm{ha}$ ) and grain with higher test weights (by 2.2 $\mathrm{kg} / \mathrm{hl}$ ). Thousand kernel weights (TKWs) were increased by $1.9 \mathrm{~g}$ and grain was 
found to have DON concentrations that averaged $5.6 \mu \mathrm{g} / \mathrm{g}$ (compared to $9.1 \mu \mathrm{g} / \mathrm{g}$ for untreated grain). These parameters are reported to be directly affected by FHB (35). Differences between treated and untreated plots were greatest in susceptible cvs. Norm and Vance compared to those of MR (36) cv. Marshall.

The purpose of this study was to evaluate the efficacy of selected chemical families, including ethylene bisdithiocarbamate (mancozeb), on the yield and quality parameters of spring wheat. Benomyl and tebuconazole were included in these experiments because these fungicides have been reported to reduce the severity of FHB-induced losses in Europe $(13,20$, 31,33), and South America $(11,24)$. Rates in excess of those federally registered for leaf disease control were examined and application timings were focused on growth stages at or near anthesis (Feekes growth stage 10.5). In 1995 and 1996, lower rates of selected fungicides, including propiconazole, were tested on wheat and experiments were established on a susceptible 6-row malting barley cultivar. Propiconazole has been shown to reduce FHB in some studies $(10,19)$. Additional experiments examined the efficacy of fungicides with potentially reduced or zero oncogenic risk (Class $\mathrm{E}$ oncogens), including azoxystrobin and fludioxonil. The influence of partial resistance (moderate susceptibility) in spring wheat cultivars on treatment benefits was also evaluated.

\section{MATERIALS AND METHODS}

The efficacy of heading applications of fungicides was evaluated in 15 field experiments conducted between 1994 and 1997. Susceptible HRSW cv. Norm $(5,15,18)$ was used in initial experimentation and MS HRSW cv. $2375(5,15,18)$ was used in later studies. Norm was widely grown in Minnesota prior to the FHB epidemics of 1993 to 1994 (18). Norm is less susceptible than other HRSW cultivars to foliage diseases such as tan spot (5) and is resistant to stem rust and leaf rust (5). The reaction of spring wheat cultivars to Septoria diseases in Minnesota is not well characterized. Diseases such as Stagonospora leaf and glume blotch (caused by Stagonospora nodorum (Berk.) Castellani \& E. G. Germano [Phaeosphaeria nodorum (E. Muller) Hedjaroude]) and Septoria tritici blotch (caused by Septoria tritici Roberge in Desmaz. [Mycosphaerella graminicola (Fuckel) J. Schröt. in Cohn]) produce limited symptoms on Norm, whereas Stagonospora leaf blotch (caused by Stagonospora aveneae (Bissett) f. sp. triticea $\mathrm{T}$. Johnson [Phaeosphaeria avenaria (G. F. Weber) O. Eriksoon f. sp. triticea T. Johnson]) is occasionally damaging (R. K. Jones, personal observations and unpublished data). Norm was chosen for these studies to reduce interactions with these leaf and head diseases. Cv. 2375 is less susceptible than
Norm to FHB (5) and exhibits an MS reaction to rachis colonization (Type 2 resistance; 28). However, 2375 is susceptible to Stagonospora leaf and glume blotch (15). Despite this susceptibility to leaf and glume blotch, 2375 was widely adopted by Minnesota wheat growers from 1994 to 1997.

Spray applications. Treatments were applied in water at a rate of 85 liters/ha with a $\mathrm{CO}_{2}$ backpack sprayer (R\&D Sprayers, Inc. Opelousas, LA) using TeeJet 8002 flat-fan nozzles on $0.5-\mathrm{m}$ spacing at $275 \mathrm{kPa}$ (40 psi). Boom height was adjusted to optimize spray pattern overlap at head height. Treatment controls were sprayed with water. Treatments were applied at Feekes growth stage 10.5.1 (unless otherwise stated). In wheat, stage 10.5.1 was considered reached after head emergence was complete and $50 \%$ of primary (nontiller) spikes were exhibiting anthers on 15 to $25 \%$ of the spikelets (29). In barley, Feekes growth stage 10.5.1 was considered reached after $100 \%$ spike emergence and peduncle elongation was complete (2). Unless otherwise stated, treatments were arranged in a randomized complete block (RCB) design with either six or four replications. Individual plots were 3.1 by 7.6 $\mathrm{m}$ and alleys between blocks were $1.5 \mathrm{~m}$ wide. Alleys were established by mowing through solid plantings immediately prior to applying fungicide treatments. The sprayed area of the plots was 2.7 by $7.6 \mathrm{~m}$, and 1.5by-7.6- $\mathrm{m}$ areas of the plot were harvested by combine.

Disease assessments. FHB was assessed at Feekes growth stage 11.2 (soft dough stage) approximately 28 days after the beginning of anthesis. Sixty consecutively sampled, primary spikes were assessed from $1 \mathrm{~m}$ of row at three evenly spaced intervals in each plot (180 total). In the 1994 trials, spikes were removed (excised $1 \mathrm{~cm}$ below the basal spikelet), assessed for $\mathrm{FHB}$, dried in forced air $\left(72 \mathrm{~h}\right.$ at $\left.95^{\circ} \mathrm{C}\right)$, and weighed. In trials in 1995 to 1997 , spikes were assessed for FHB while still attached to plants. Spikes of wheat and barley were rated on a 0 -to- 6 scale as a modification of the system of Chelkowski (8), where rating class $0=$ no symptoms, 1 $=$ one spikelet damaged $(6.25 \%$ disease $), 2$ $=2$ to 3 spikelets damaged $(12.5 \%$ disease), $3=4$ to 5 spikelets damaged (31.25\% disease), $4=8$ spikelets damaged ( $50 \%$ disease), $5=12$ spikelets damaged ( $75 \%$ disease), and $6=15$ or more spikelets damaged (93.75\% disease). Disease severity was calculated by multiplying the frequency of spikes in each of the 0 to 6 rating classes by the disease severity for that class $(0.0$ to 0.9375$)$. These values were summed, and converted to a percentage for analysis. Leaf diseases were evaluated from plants in the same three 1-m row sections sampled for FHB by using a 0-to9 Saari scale (27) as modified by Hosford (12), where $0=$ disease-free and $9=>50 \%$ of upper, middle, and lower leaves exhibiting symptoms.

Post-harvest assessments. At harvest, wheat grain was dried to a uniform moisture content (approximately 12.5\%) and cleaned with standard screens to remove straw, chaff, and weed seeds. The cleaned grain was weighed and yield calculated. The grain volume was then reduced to approximately $500 \mathrm{~g}$ with a 16-chute riffledivider with $2.5-\mathrm{cm}$ openings (Model H3987; Humbolt Testing Equipment, Norridge, IL). Test weight (TW) and, subsequently, TKW were determined on this sample using standard methods (4). The remaining portion of the TW sample was reduced to $100 \mathrm{~g}$ using a Boerner sample divider (Model 34; Seedburo Equipment Company, Chicago). The percent visually scabby grain (\% VSK) was determined for this sample using previously described methods (18). In barley, samples were dried, cleaned, and weighed as described for wheat. The percent plump kernels (\% plump) was determined for barley samples as the weight proportion of $250 \mathrm{~g}$ that would not pass through a 2.38-by19.05-mm slotted screen (4). Kernel discoloration (KD) was rated on a 1-to-5 scale ( $1=$ bright and $5=$ severely discolored $)$ using a representative 100-g subsample according to the method of Miles et al. (22). In all studies, DON concentration was quantified as the tri-methyl silyl derivative with gas chromatography using the most representative subsample of grain (VSK subsamples for wheat and KD subsamples for barley) with methods that have been previously reported (18).

High-rate and multiple-application studies on a susceptible cultivar. High rates and multiple applications of fungicides were tested for their ability to reduce FHB in trials conducted in 1994 and 1995. In 1994, two experiments (one nonirrigated, one irrigated) were established at the West Central Experiment Station (WCES) in Morris, MN, and one (nonirrigated) experiment was established at the Northwest Experiment Station (NWES) in Crookston, MN. Tests were conducted on susceptible cv. Norm. Six treatments replicated six times were arranged in a Latin square design. Treatments were: (i) benomyl (Benlate 50WP) at $560 \mathrm{~g}$ a.i./ha, (ii) iprodione (Rovral 4F) at $560 \mathrm{~g}$ a.i./ha, (iii) mancozeb (Dithane 75DF) at 1,680 $\mathrm{g}$ a.i./ha, (iv) tebuconazole (Folicur 3.6F) at $190 \mathrm{~g}$ a.i./ha, (v) triphenyl tin hydroxide (Super Tin 4L) at $190 \mathrm{~g}$ a.i./ha, and (vi) no fungicide (Table 1). Fungicides were applied twice, at Feekes growth stage 10.5 and again at Feekes 11.0 (14 days later). Latron CS-7 spreader binder (the alkylaryl polyethoxylate and sodium salt of alkylsulfonatedalkylate) was added to treatments 1 and 3 at $120 \mathrm{ml} / \mathrm{ha}$ as per the manufacturer's recommendations.

At Morris, one of the two sites was established with a mist irrigation system. The 
system consisted of spinner nozzles (Micro-Bird II; Rain Bird Sprinkler Mfg. Corp., Glendora, CA) mounted at 6.1-m spacing on 1-m risers staggered in relation to adjacent irrigation lines and spaced 6.1 $\mathrm{m}$ apart. The spray mist from each nozzle has an effective diameter of $8.4 \mathrm{~m}$. The output of the mist irrigation system provided the equivalent of $0.8 \mathrm{~mm}$ of water for $10 \mathrm{~min}$ at the beginning of each hour. Mist irrigation commenced at the beginning of head emergence (Feekes growth stage 10.0), continued for 28 days, and was terminated at the soft dough stage (Feekes growth stage 11.2). The mist system was turned off $24 \mathrm{~h}$ prior to and remained off for $24 \mathrm{~h}$ after fungicide treatments were applied. A nonirrigated trial was also established at Morris. This trial was located about $500 \mathrm{~m}$ from the trial that was misted. Corn (Zea mays L.) was planted the previous season at both Morris sites and corn residue (10 to $15 \%$ surface cover) was uniformly available as a source of inoculum. At Crookston in 1994, the previous crop was barley. In 1995, the high-rate study was modified and repeated at Morris without irrigation. New comparisons included one versus two applications of each fungicide treatment: (i) Feekes 10.5 versus (ii) Feekes 10.5 plus Feekes 11.0 (14 days later). Treatments (Table 2) were arranged in an RCB design. Triphenyltin hydroxide was not included in the 1995 trial. The relationships between the assessment variables collected in this study (Table 3) were examined using Pearson correlation coefficients.

Low-rate and single-application studies on a susceptible cultivar. The efficacy of lower rates of selected fungicides and fungicide tank mixes were evaluated in trials conducted at Crookston in 1995 and 1996. Experiments were established on susceptible cv. Norm. Ten treatments replicated six times were arranged in an RCB design (Table 4). Treatments were: (i) benomyl at $280 \mathrm{~g}$ a.i./ha plus mancozeb at $840 \mathrm{~g} \mathrm{a.i} / \mathrm{ha}$, (ii) benomyl at $280 \mathrm{~g}$ a.i./ha plus tebuconazole at $126 \mathrm{~g}$ a.i./ha, (iii) carbendazim at $133 \mathrm{~g}$ a.i./ha plus tebuconazole at $167 \mathrm{~g}$ a.i./ha (Libero SC300), (iv) difenozonazole (Dividend 3.5EC) at $122 \mathrm{~g}$ a.i./ha, (v) difenoconazole at $245 \mathrm{~g}$ a.i./ha, (vi) fenbuconazole (Govern 75WP) at $210 \mathrm{~g}$ a.i./ha, (vii) fenbuconazole at 420 $\mathrm{g}$ a.i./ha, (viii) fludioxonil (Maxim 50WP) at $560 \mathrm{~g}$ a.i./ha, (ix) mancozeb at $1,680 \mathrm{~g}$ a.i./ha, and $(\mathrm{x})$ no fungicide. Fungicides were applied at Feekes 10.5.1.

Several registered and unregistered fungicides were evaluated in trials conducted under mist irrigation at Morris in 1995 and 1996. Experiments were established on susceptible cv. Norm. Ten treatments replicated six times were arranged in an RCB design (Table 5). Treatments were: (i) azoxystrobin (Quadris 80WG) at $224 \mathrm{~g}$ a.i./ha, (ii) azoxystrobin at $448 \mathrm{~g}$ a.i./ha, (iii) benomyl at $560 \mathrm{~g}$ a.i./ha, (iv) benomyl at $280 \mathrm{~g}$ a.i./ha plus mancozeb at $840 \mathrm{~g}$ a.i./ha, (v) benomyl at $280 \mathrm{~g}$ a.i./ha plus propiconazole (Tilt 3.6EC) at $126 \mathrm{~g}$ a.i./ha, (vi) benomyl at $280 \mathrm{~g}$ a.i./ha plus tebuconazole at $126 \mathrm{~g}$ a.i./ha, (vii) fludioxonil at $560 \mathrm{~g}$ a.i./ha, (viii) propiconazole at 126 $\mathrm{g}$ a.i./ha, (ix) tebuconazole at $126 \mathrm{~g}$ a.i./ha, and $(\mathrm{x})$ no fungicide. Fungicides were applied at Feekes 10.5.1. In both years, misting was applied for 20 min every $4 \mathrm{~h}$ beginning 7 days prior to anthesis and

Table 1. Effects of fungicides on Fusarium head blight (FHB), leaf disease indices, head weight, yield, and post-harvest quality parameters from the FHBsusceptible spring wheat cultivar Norm at three locations in1994

\begin{tabular}{|c|c|c|c|c|c|c|c|c|c|}
\hline Treatment (a.i./ha) ${ }^{\mathrm{a}}$ & $\underset{(\%)^{\mathbf{b}}}{\text { FHB I }}$ & $\begin{array}{c}\text { FHB S } \\
(\%)^{\mathbf{b}}\end{array}$ & $\begin{array}{c}\text { Leaf DI } \\
(0-9)^{c}\end{array}$ & $\begin{array}{c}\text { Head } \\
\text { weight }(g)^{d}\end{array}$ & $\begin{array}{c}\text { Yield } \\
\text { (kg/ha) }\end{array}$ & $\begin{array}{l}\text { Test weight } \\
\text { (kg/hl) }\end{array}$ & TKW $(g)^{\mathbf{e}}$ & $\operatorname{VSK}(\%)^{\mathbf{f}}$ & $\begin{array}{c}\text { DON } \\
(\mu \mathrm{g} / \mathrm{g}) \mathrm{g}\end{array}$ \\
\hline Benomyl, $560 \mathrm{~g}$ & 66 & 22 & 6.0 & 0.853 & 2,915 & 68.3 & 26.0 & 10.5 & 5.3 \\
\hline Iprodione, $560 \mathrm{~g}$ & 81 & 37 & 6.7 & 0.783 & 2,299 & 64.7 & 24.3 & 22.8 & 14.2 \\
\hline Mancozeb, $1,680 \mathrm{~g}$ & 72 & 29 & 5.9 & 0.826 & 2,582 & 66.0 & 24.6 & 16.4 & 11.2 \\
\hline Tebuconazole, $190 \mathrm{~g}$ & 65 & 22 & 5.3 & 0.873 & 3,032 & 69.5 & 27.3 & 11.3 & 5.2 \\
\hline Triphenyltin, $190 \mathrm{~g}$ & 76 & 31 & 6.3 & 0.808 & 2,466 & 65.3 & 24.1 & 17.0 & 9.9 \\
\hline No fungicide & 79 & 37 & 6.9 & 0.799 & 2,332 & 64.5 & 23.5 & 18.9 & 11.5 \\
\hline $\operatorname{LSD}(P=0.05)$ & 8 & 5 & 0.4 & 0.043 & 387 & 2.1 & 1.1 & 2.8 & 2.6 \\
\hline
\end{tabular}

${ }^{a}$ Treatments applied at Feekes growth stage 10.5 and Feekes growth stage 11.0 (14 days later). Latron CS-7 was added to benomyl and mancozeb treatments at $120 \mathrm{ml} / \mathrm{ha}$ as per the manufacturer's recommendations. $\mathrm{LSD}=$ least significant difference.

${ }^{\mathrm{b}}$ FHB incidence (I) and severity (S) based on assessment of 3,240 heads.

${ }^{c}$ Leaf disease index assessed on a 0-to-9 Hosford scale (12) at Feekes growth stage 11.2.

${ }^{d}$ Mean weight per head of 3,240 heads excised at Feekes growth stage 11.2.

e Thousand kernel weight.

${ }^{\mathrm{f}}$ Visually scabby kernels in harvested grain.

$\mathrm{g}$ Deoxynivalenol concentration.

Table 2. Effects of fungicides on Fusarium head blight (FHB), leaf disease indices, yield, and post-harvest quality parameters from the FHB-susceptible, spring wheat cultivar Norm sprayed with fungicides at different timings at the West Central Experiment Station in Morris, MN, in 1995

\begin{tabular}{|c|c|c|c|c|c|c|c|c|c|}
\hline Treatment $^{\mathrm{a}}$ & $\begin{array}{c}\text { Application } \\
\text { timings }\end{array}$ & FHB I $(\%)^{\mathbf{b}}$ & FHB $S(\%)^{b}$ & $\begin{array}{c}\text { Leaf DI } \\
(0-9)^{\mathrm{c}}\end{array}$ & $\begin{array}{c}\text { Yield } \\
(\mathrm{kg} / \mathrm{ha})\end{array}$ & $\begin{array}{l}\text { Test weight } \\
(\mathrm{kg} / \mathrm{hl})\end{array}$ & TKW $(g)^{d}$ & $\operatorname{VSK}(\%)^{\mathrm{e}}$ & DON $(\mu \mathrm{g} / \mathrm{g})^{\mathrm{f}}$ \\
\hline Benomyl & 10.5 & 26 & 5 & 5.5 & 3,657 & 71.6 & 32.1 & 7.5 & 1.3 \\
\hline Benomyl & $10.5 / 11.0$ & 23 & 4 & 5.6 & 3,921 & 73.0 & 32.5 & 7.5 & 1.5 \\
\hline Iprodione & 10.5 & 48 & 9 & 5.9 & 3,189 & 65.6 & 28.6 & 20.0 & 5.8 \\
\hline Iprodione & $10.5 / 11.0$ & 46 & 8 & 6.1 & 3,155 & 66.6 & 27.6 & 22.5 & 6.0 \\
\hline Mancozeb & 10.5 & 48 & 10 & 5.4 & 3,400 & 68.2 & 29.7 & 17.5 & 5.6 \\
\hline Mancozeb & $10.5 / 11.0$ & 44 & 8 & 4.7 & 3,442 & 67.3 & 28.9 & 17.5 & 3.9 \\
\hline Tebuconazole & 10.5 & 35 & 5 & 5.0 & 3,868 & 70.9 & 31.6 & 12.5 & 2.5 \\
\hline Tebuconazole & $10.5 / 11.0$ & 29 & 4 & 5.2 & 3,941 & 72.7 & 32.7 & 7.5 & 2.0 \\
\hline No fungicide & $\ldots$ & 52 & 10 & 6.4 & 3,090 & 64.7 & 27.1 & 20.0 & 6.1 \\
\hline $\operatorname{LSD}(P=0.05)$ & $\ldots$ & 10 & 2 & 0.8 & 295 & 3.3 & 2.0 & 6.8 & 1.3 \\
\hline
\end{tabular}

${ }^{a}$ Treatments applied at Feekes 10.5 at the following rates (a.i./ha): benomyl, $560 \mathrm{~g}$; iprodione, $560 \mathrm{~g}$; mancozeb, 1,680 g; and tebuconazole, $190 \mathrm{~g}$. Treatments $3,5,7$, and 9 were resprayed with the same chemical and rate at Feekes 11.0 (14 days later). Latron CS-7 at 120 ml/ha was added to benomyl and mancozeb treatments as per the manufacturer's recommendations. LSD = least significant difference.

${ }^{\mathrm{b}}$ FHB incidence (I) and severity (S) based on assessment of 1,080 heads.

${ }^{\mathrm{c}}$ Leaf disease index assessed on a 0-to-9 Hosford scale (12) at Feekes growth stage 11.2.

d Thousand kernel weight.

e Visually scabby kernels in harvested grain.

${ }^{\mathrm{f}}$ Deoxynivalenol concentration. 
continued for 21 days after anthesis. Misting was turned off $24 \mathrm{~h}$ prior to and remained off for $24 \mathrm{~h}$ after treatments were applied.

Low-rate and single-application studies on an MS cultivar. The effect of fungicides on the development of FHB in MS cv. 2375 was evaluated in four experiments conducted in 1995 and 1997. Four treatments replicated four times were arranged in an RCB design (Table 6). Treatments were (i) azoxystrobin at $224 \mathrm{~g}$ a.i./ha, (ii) propiconazole at $126 \mathrm{~g}$ a.i./ha, (iii) tebuconazole at $126 \mathrm{~g}$ a.i./ha, and (iv) no fungicide. Fungicides were applied at Feekes growth stage 10.5.1. Two of four experiments were established into the previous year's wheat residue; in the other two experiments, nonhosts were the previous crops.
Cultivar-fungicide interaction study. The effect of fungicides on seven spring wheat cultivars with differing susceptibilities to FHB was evaluated in 1995 at Crookston (Table 7). The experiment was established into wheat residue. Cultivars tested were 2375 (MS), Butte 86 (MS), Grandin (MS), Marshall (MR), Norm (S), Sharp (MS), and Verde (MS). Whole plots were 4.9 by $7.0 \mathrm{~m}$, arranged in an RCB

Table 3. Pearson correlation coefficients for correlations of Fusarium head blight incidence (FHBI), FHB severity (FHBS), leaf disease index (LDI), yield (YLD), test weight (TWT), thousand kernel weight (TKW), percent visually scabby kernels (VSK), and deoxynivalenol (DON) concentration at the West Central Experiment Station in Morris, MN, in 1995ª

\begin{tabular}{|c|c|c|c|c|c|c|c|c|}
\hline \multirow[b]{2}{*}{ Variable } & \multicolumn{8}{|c|}{ Variable } \\
\hline & FHBI & FHBS & LDI & YLD & TWT & TKW & VSK & DON \\
\hline FHBI & $\ldots$ & 0.88 & 0.52 & -0.64 & -0.60 & -0.60 & 0.69 & 0.70 \\
\hline FHBS & $\ldots$ & $\ldots$ & 0.50 & -0.65 & -0.61 & -0.60 & 0.73 & 0.73 \\
\hline LDI & $\ldots$ & $\ldots$ & $\ldots$ & -0.49 & -0.46 & 0.41 & 0.46 & 0.46 \\
\hline YLD & $\ldots$ & $\ldots$ & $\ldots$ & $\ldots$ & 0.72 & 0.75 & -0.73 & -0.64 \\
\hline TWT & $\ldots$ & $\ldots$ & $\ldots$ & $\ldots$ & $\ldots$ & 0.78 & -0.74 & -0.65 \\
\hline TKW & $\cdots$ & $\ldots$ & 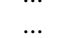 & $\ldots$ & $\cdots$ & $\ldots$ & -0.77 & -0.70 \\
\hline VSK & $\ldots$ & $\ldots$ & $\ldots$ & $\ldots$ & $\ldots$ & $\ldots$ & $\ldots$ & 0.86 \\
\hline DON & $\ldots$ & $\ldots$ & $\ldots$ & $\ldots$ & $\ldots$ & $\ldots$ & $\ldots$ & $\ldots$ \\
\hline
\end{tabular}

a All correlation coefficients shown are significant at $P<0.01$.

Table 4. Disease assessments and grain quality parameters from the Fusarium head blight (FHB)-susceptible, spring wheat cultivar Norm at the Northwest Experiment Station in Crookston, MN in 1995 to 1996

\begin{tabular}{|c|c|c|c|c|c|c|c|}
\hline Treatment, a.i./ha & $\begin{array}{c}\text { FHB I } \\
(\%)^{b}\end{array}$ & $\begin{array}{c}\text { FHB S } \\
(\%)^{b}\end{array}$ & $\begin{array}{c}\text { Leaf DI } \\
(0-9)^{c}\end{array}$ & Yield (kg/ha) & $\begin{array}{c}\text { Test weight } \\
(\mathrm{kg} / \mathrm{hl})\end{array}$ & $\operatorname{VSK}(\%)^{\mathbf{d}}$ & $\begin{array}{c}\text { DON } \\
(\mu g / g)^{e}\end{array}$ \\
\hline Benomyl, $280 \mathrm{~g}+$ Mancozeb, $840 \mathrm{~g}$ & 65 & 16 & 5.2 & 3,139 & 69.3 & 20.2 & 5.7 \\
\hline Benomyl, $280 \mathrm{~g}$ + Tebuconazole, $126 \mathrm{~g}$ & 66 & 17 & 5.2 & 3,410 & 70.1 & 17.8 & 5.0 \\
\hline Carbendazim, $133 \mathrm{~g}+$ Tebuconazole, $167 \mathrm{~g}$ & 68 & 18 & 5.1 & 3,228 & 69.0 & 21.1 & 5.1 \\
\hline Difenoconazole, $122 \mathrm{~g}$ & 76 & 23 & 5.3 & 2,880 & 67.9 & 24.0 & 8.6 \\
\hline Difenoconazole, $245 \mathrm{~g}$ & 70 & 21 & 4.9 & 3,047 & 68.0 & 24.0 & 8.7 \\
\hline Fenbuconazole, $210 \mathrm{~g}$ & 79 & 27 & 5.5 & 2,812 & 67.0 & 28.3 & 9.2 \\
\hline Fenbuconazole, $420 \mathrm{~g}$ & 75 & 24 & 5.5 & 2,899 & 67.1 & 27.0 & 8.8 \\
\hline Fludioxonil, $560 \mathrm{~g}$ & 70 & 22 & 6.1 & 3,105 & 69.1 & 22.2 & 6.3 \\
\hline Mancozeb, 1,680 g & 75 & 24 & 5.7 & 2,946 & 67.3 & 26.3 & 8.8 \\
\hline No fungicide & 81 & 28 & 6.9 & 2,690 & 66.1 & 29.0 & 10.2 \\
\hline $\operatorname{LSD}(P=0.05)$ & 8 & 5 & 0.5 & 290 & 1.8 & 5.1 & 1.8 \\
\hline
\end{tabular}

a Treatments applied at Feekes 10.5 .1 (15 to 25\% anthesis). Latron CS-7 at $120 \mathrm{ml} / \mathrm{ha}$ was added to benomyl and mancozeb treatments as per manufacturer's recommendations. LSD = least significant difference.

${ }^{\mathrm{b}}$ FHB incidence (I) and severity (S) based on assessment of 1,080 heads.

${ }^{c}$ Leaf disease index assessed on a 0-to-9 Hosford scale (12) at Feekes growth stage 11.2.

${ }^{\mathrm{d}}$ Visually scabby kernels in harvested grain.

e Deoxynivalenol concentration.

Table 5. Disease assessments and grain quality parameters from the Fusarium head blight (FHB)-susceptible, spring wheat cultivar Norm at the West Central Experiment Station in Morris, MN, in 1995 to 1996

\begin{tabular}{|c|c|c|c|c|c|c|c|}
\hline Treatment, a.i./ha ${ }^{\mathrm{a}}$ & FHB I $(\%)^{\mathbf{b}}$ & FHB S $(\%)^{b}$ & $\begin{array}{c}\text { Leaf DI } \\
(0-9)^{c}\end{array}$ & Yield (kg/ha) & $\begin{array}{c}\text { Test weight } \\
(\mathrm{kg} / \mathrm{hl})\end{array}$ & $\operatorname{VSK}(\%)^{\mathbf{d}}$ & $\begin{array}{c}\text { DON } \\
(\mu \mathrm{g} / \mathrm{g})^{\mathrm{e}}\end{array}$ \\
\hline Azoxystrobin, $224 \mathrm{~g}$ & 75 & 22 & 4.8 & 2,668 & 60.1 & 40.8 & 18.5 \\
\hline Azoxystrobin, $448 \mathrm{~g}$ & 68 & 18 & 4.5 & 2,880 & 63.0 & 36.3 & 15.5 \\
\hline Benomyl, $560 \mathrm{~g}$ & 54 & 13 & 5.0 & 2,994 & 63.5 & 30.0 & 12.1 \\
\hline Benomyl, $280 \mathrm{~g}+$ Mancozeb, $840 \mathrm{~g}$ & 58 & 15 & 4.8 & 2,852 & 63.7 & 30.9 & 10.0 \\
\hline Benomyl, $280 \mathrm{~g}+$ Propiconazole, $126 \mathrm{~g}$ & 58 & 14 & 4.0 & 2,989 & 64.4 & 30.4 & 11.6 \\
\hline Benomyl, $280 \mathrm{~g}+$ Tebuconazole, $126 \mathrm{~g}$ & 60 & 16 & 4.4 & 3,016 & 63.3 & 30.3 & 11.8 \\
\hline Fludioxonil, $560 \mathrm{~g}$ & 62 & 16 & 4.4 & 2,923 & 63.5 & 34.6 & 13.3 \\
\hline Propiconazole, $126 \mathrm{~g}$ & 62 & 16 & 3.8 & 2,874 & 62.8 & 35.0 & 13.6 \\
\hline Tebuconazole, $126 \mathrm{~g}$ & 60 & 15 & 3.9 & 2,906 & 63.5 & 33.1 & 13.1 \\
\hline No fungicide & 85 & 29 & 5.6 & 2,396 & 59.2 & 44.2 & 18.3 \\
\hline $\operatorname{LSD}(P=0.05)$ & 8 & 4 & 0.7 & 196 & 1.8 & 4.2 & 2.8 \\
\hline
\end{tabular}

a Treatments applied at Feekes 10.5 .1 (15 to $25 \%$ anthesis). Latron CS-7 at $120 \mathrm{ml} / \mathrm{ha}$ was added to benomyl and mancozeb treatments as per the manufacturer's recommendations. LSD = least significant difference.

b FHB incidence (I) and severity (S) based on assessment of 1,080 heads.

${ }^{\mathrm{c}}$ Leaf disease index assessed on a 0-to-9 Hosford scale (12) at Feekes growth stage 11.2.

$\mathrm{d}$ Visually scabby kernels in harvested grain.

e Deoxynivalenol concentration. 
design, and replicated four times. Alleys between blocks were $1.5 \mathrm{~m}$ wide. Subplots of each cultivar were sprayed or not sprayed with a mixture of benomyl (280 g a.i./ha) plus propiconazole (126 g a.i./ha) at Feekes 10.5.1, based on the date when a particular cultivar reached 15 to $25 \%$ anthesis. These growth stages were reached between 2 and 8 July in 1995. The sprayed area of the plots were 2.4 by $7.6 \mathrm{~m}$ At harvest, plot lengths were trimmed to $6.1 \mathrm{~m}$ and 1.5 -by-6.1-m areas of the plot were harvested by combine.

Barley studies. The effect of fungicides on FHB development in the susceptible 6row malting barley cultivar Stander was evaluated in trials at Crookston in 1995 and 1996. Ten treatments replicated six times were arranged in an RCB design (Table 8). Treatments were (i) benomyl at $560 \mathrm{~g}$ a.i./ha, (ii) benomyl at $280 \mathrm{~g}$ a.i./ha plus mancozeb at $840 \mathrm{~g}$ a.i/ha, (iii) benomyl at $280 \mathrm{~g}$ a.i./ha plus propiconazole at $126 \mathrm{~g}$ a.i./ha, (iv) difenoconazole at $122 \mathrm{~g}$ a.i./ha, (v) fenbuconazole at $210 \mathrm{~g}$ a.i./ha, (vi) fludioxonil at $560 \mathrm{~g}$ a.i./ha, (vii) mancozeb at 1,680 $\mathrm{g}$ a.i./ha, (viii) propicona- zole at $126 \mathrm{~g}$ a.i./ha, (ix) tebuconazole at $126 \mathrm{~g}$ a.i./ha, and (x) no fungicide. Fungicides were applied at Feekes growth stage 10.5.1.

Statistical analysis of field trial results. Data from field trials were examined using analysis of variance (ANOVA) to evaluate differences in disease incidence, disease severity, yield and post-harvest parameters. Computations were performed using the General AOV procedure of Statistix for Windows (version 2.0; Analytical Software, Tallahassee, FL). Mean separations were determined using the least significant difference $(P=0.05)$. Paired $t$ tests were used to examine treatment differences in the cultivar-fungicide interaction study.

Fungicidal activity in vitro. The effect of selected fungicides on the radial growth rate of isolates of $F$. graminearum was compared. Dilutions of fungicides were prepared by mixing appropriate amounts of each fungicide (based on active ingredient) in $5 \mathrm{ml}$ of $95 \%$ ethanol. Equal volumes of ethanol containing diluted fungicides were added to sterile, cool potato-dextrose agar (PDA, Difco Laboratories, Detroit) to achieve concentrations of $0.001,0.01,0.1$, $1.0,10,100$, and $1,000 \mathrm{mg}$ a.i./liter of the following active ingredients: (i) benomyl, (ii) fludioxonil, (iii) mancozeb, and (iv) tebuconazole. A zero-concentration treatment was prepared for each isolate-fungicide combination and contained $1 \%$ ( $\mathrm{vol} / \mathrm{vol}$ ) of $95 \%$ ethanol to ensure equivalent ethanol concentrations in each treatment. Fungicide-amended PDA was dispensed aseptically into 9-cm-diameter plastic petri dishes (about $20 \mathrm{ml}$ per dish).

Mycelial plugs ( $7 \mathrm{~mm}$ in diameter) were cut from the margins of actively growing cultures of three isolates (SP4, M6, and CD1) and inverted in the centers of fungicide-amended and unamended PDA plates. There were six replicate plates for each isolate-fungicide combination. Plates were incubated for $72 \mathrm{~h}$ at $25^{\circ} \mathrm{C}$ in the dark. Colony diameters were measured twice (random and at right angles) and adjusted for the diameter of the plug. The effective fungicide concentration was determined by interpolating plots of growth inhibition probits for concentrations that give $50 \%$ $\left(\mathrm{ED}_{50}\right)$ or $90 \%\left(\mathrm{ED}_{90}\right)$ growth inhibition for

Table 6. Disease assessments and grain quality parameters from the Fusarium head blight (FHB)-moderately susceptible, spring wheat cultivar 2375 at three locations in 1995 to 1997

\begin{tabular}{|c|c|c|c|c|c|c|c|}
\hline Treatment, a.i./ha ${ }^{a}$ & FHB I $(\%)^{b}$ & FHB S $(\%)^{b}$ & Leaf DI $(0-9)^{c}$ & Yield (kg/ha) & Test weight (kg/hl) & $\operatorname{VSK}(\%)^{\mathrm{d}}$ & DON $(\mu \mathrm{g} / \mathrm{g})^{\mathrm{e}}$ \\
\hline Azoxystrobin, $224 \mathrm{~g}$ & 69 & 22 & 6.7 & 3,265 & 74.5 & 5.7 & 2.5 \\
\hline Propiconazole, $126 \mathrm{~g}$ & 65 & 18 & 6.1 & 3,424 & 74.4 & 4.6 & 2.1 \\
\hline Tebuconazole, $126 \mathrm{~g}$ & 61 & 16 & 6.4 & 3,292 & 75.0 & 3.8 & 1.6 \\
\hline No fungicide & 78 & 26 & 7.9 & 2,797 & 72.4 & 9.6 & 3.8 \\
\hline $\operatorname{LSD}(P=0.05)$ & 4 & 3 & 0.6 & 263 & 0.9 & 2.1 & 0.7 \\
\hline
\end{tabular}

a Treatments applied at Feekes 10.5.1 (15 to 25\% anthesis). LSD = least significant difference.

${ }^{\mathrm{b}}$ FHB incidence (I) and severity (S) based on assessment of 2,160 heads.

${ }^{c}$ Leaf disease index assessed on a 0-to-9 Hosford scale (12) at Feekes growth stage 11.2.

${ }^{\mathrm{d}}$ Visually scabby kernels in harvested grain.

e Deoxynivalenol concentration.

Table 7. Disease assessments and grain quality parameters from seven spring wheat cultivars at the Northwest Experiment Station in Crookston, MN in $1995^{\mathrm{a}}$

\begin{tabular}{|c|c|c|c|c|c|c|c|c|}
\hline Cultivar & $\begin{array}{l}\text { Fungicide } \\
\text { treated }\end{array}$ & FHB I $(\%)^{b}$ & FHB S $(\%)^{b}$ & $\begin{array}{l}\text { Leaf yield DI } \\
(0-9)^{\mathrm{c}}\end{array}$ & Yield (kg/ha) & $\begin{array}{l}\text { Test weight } \\
(\mathrm{kg} / \mathrm{hl})\end{array}$ & $\operatorname{VSK}(\%)^{\mathrm{d}}$ & DON $(\mu \mathrm{g} / \mathrm{g})^{\mathrm{e}}$ \\
\hline \multirow[t]{2}{*}{2375} & + & 63 & $12 * *$ & $5.3^{*}$ & 3,414 & 71.7 & 4.8 & 1.8 \\
\hline & - & 70 & 17 & 7.2 & 3,199 & 71.2 & 7.0 & 2.7 \\
\hline \multirow{2}{*}{ Butte 86} & + & $52 *$ & $10 * *$ & $5.7 *$ & $3,541 *$ & 71.9 & 7.0 & 1.7 \\
\hline & - & 63 & 14 & 7.0 & 3,138 & 71.2 & 9.3 & 2.0 \\
\hline \multirow[t]{2}{*}{ Grandin } & + & 62 & $10^{*}$ & $4.8^{* *}$ & $3,407 * *$ & 72.1 & $6.5^{*}$ & $2.2 *$ \\
\hline & - & 72 & 16 & 7.9 & 2,849 & 70.9 & 18.8 & 3.1 \\
\hline \multirow[t]{2}{*}{ Marshall } & + & 53 & $8^{*}$ & $5.3 * *$ & $3,636^{* *}$ & 68.5 & 9.8 & 2.0 \\
\hline & - & 62 & 10 & 7.0 & 3,152 & 68.3 & 14.5 & 2.0 \\
\hline \multirow[t]{2}{*}{ Norm } & + & $75^{*}$ & $27 * *$ & 4.3 & $2,829 *$ & 62.0 & 41.3 & 8.3 \\
\hline & - & 86 & 40 & 4.8 & 2,379 & 60.5 & 47.5 & 9.0 \\
\hline \multirow[t]{2}{*}{ Sharp } & + & $52 *$ & $10 * *$ & $5.9 *$ & $3,562 * *$ & $74.5^{*}$ & 4.0 & 1.4 \\
\hline & - & 66 & 18 & 6.8 & 3,111 & 72.8 & 5.0 & 2.0 \\
\hline \multirow[t]{2}{*}{ Verde } & + & $48^{*}$ & $10^{*}$ & $4.7 *$ & $3,528^{*}$ & $69.5^{*}$ & 20.0 & 1.8 \\
\hline & - & 66 & 17 & 6.3 & 3,064 & 68.2 & 26.3 & 2.2 \\
\hline $\operatorname{LSD}(P=0.05)$ & $\ldots$ & 9 & 5 & 0.6 & 195 & 1.4 & 6.7 & 2.0 \\
\hline
\end{tabular}

${ }^{\mathrm{a}}$ In paired plots, a tank mix of benomyl (280 g a.i./ha) + propiconazole (126 g a.i./ha) was applied at Feekes growth stage 10.5 .1 (15 to $25 \%$ anthesis) based on the anthesis date of each cultivar. For statistical purposes, analyses of variance and least significant difference (LSD) calculations were performed and can be used to compare cultivar differences among unsprayed treatments. Comparisons between sprayed versus unsprayed treatments within cultivars was done with $t$ tests. Significant differences between sprayed and unsprayed within cultivars are either denoted as $*(P<0.05)$ or $* *(P<$ $0.01)$.

${ }^{\mathrm{b}}$ Fusarium head blight (FHB) incidence (I) and severity (S) based on assessment of 720 heads.

${ }^{c}$ Leaf disease index assessed on a 0-to-9 Hosford scale (12) at Feekes growth stage 11.2.

${ }^{\mathrm{d}}$ Visually scabby kernels in harvested grain.

${ }^{\mathrm{e}}$ Deoxynivalenol concentration. 
each fungicide (17). An additional 50 isolates of $F$. graminearum, including 20 isolates used in preparing inoculum mixtures for FHB screening nurseries at the University of Minnesota (36), were tested for growth inhibition by benomyl, fludioxonil, mancozeb, and tebuconazole at 0 and 10 mg a.i./liter. All isolates tested, including SP4, M6, and CD1, were obtained from infected wheat and barley grown in Minnesota prior to $1990(37,39)$. Plates were prepared, inoculated in triplicate, and incubated as described above. Plates were examined for formation of colonies typical of $F$. graminearum after 72 and $96 \mathrm{~h}$. The adjusted growth measurements were examined using ANOVA.

Wheat seed collected from the harvested grain of field trials that had been sprayed with benomyl at 0.56 to $1.12 \mathrm{~kg}$ a.i./ha (Morris-94, Morris-95, and Morris-96) were tested for the presence of benomylresistant strains of $F$. graminearum. Seed exhibiting characteristic visual damage associated with $F$. graminearum (visually scabby kernels) were direct plated on agar amended with benomyl at $10 \mathrm{mg}$ a.i./liter. A total of 1,500 symptomatic seed (500 per year) were tested. Plates were prepared, inoculated with five surface-sterilized seed per plate, and incubated at $25^{\circ} \mathrm{C}$ in the dark. Twenty unamended plates were inoculated with a single seed in each experimental run to serve as controls. After 72 and $96 \mathrm{~h}$, plates were examined for formation of colonies of $F$. graminearum.

\section{RESULTS}

High-rate and multiple-application studies on a susceptible cultivar. FHB developed in all three experiments conducted in 1994. The incidence of FHB in unsprayed plots, expressed as the percentage of 1,080 assessed heads, was $68 \%$ in the unmisted trial at Morris, $73 \%$ in the misted trial at Morris, and 92\% at Crookston. Disease severity in unsprayed plots was 24\% (Morris, unmisted), $45 \%$ (Morris, misted), and $42 \%$ (Crookston). Mist irri- gation at Morris appeared to enhance disease severity relative to disease incidence. In a combined ANOVA, there were no significant $(P=0.05)$ treatment-site (location) interactions; therefore, data were combined for presentation. Benomyl and tebuconazole reduced FHB incidence and severity $(P=0.05)$ compared to the untreated control (Table 1). Mancozeb and triphenyltin applications reduced FHB severity $(P=0.05)$, but differences in incidence were not significant (Table 1). Iprodione was ineffective. The lower disease severity resulting from benomyl and tebuconazole applications included both a significant $(P=0.05)$ increase in the number of disease-free heads (a reduction in incidence) and a significant $(P=0.05)$ reduction in frequency for each of the higher severity class values (i.e., the number of heads in severity classes $3,4,5$, and 6) compared to plots receiving no fungicide. The weight of detached heads from treatments sprayed with benomyl or tebuconazole were significantly $(P=0.05)$ greater than the untreated control (Table 1). Benomyl, mancozeb, tebuconazole, and triphenyltin hydroxide significantly reduced $(P=0.05)$ the index of leaf diseases compared to the untreated control (Table 1). The range of differences was 5.3 to 6.9 , suggesting that leaf disease expression on Norm was limited to lower and middle leaves (12). Flag leaf damage was not extensive and the majority of the damage observed was associated with bacterial leaf streak (caused by Xanthomonas translucens (ex Jones et al., 1917), Vauterin et al., 1995), a disease not controlled by the fungicides tested in these experiments. $\mathrm{Pa}$ rameters measured at or after harvest reflected the effects of fungicide treatment on FHB; increasing yield, TW, and TKW and decreasing \% VSK and DON concentration (Table 1). Measurements of these parameters from plots sprayed with benomyl and tebuconazole were significantly different $(P=0.05)$ from the untreated check in all cases, whereas differences in these parameters between the untreated control and other fungicide treatments were not significant $(P=0.05)$.

In 1995, weather conditions at Morris were less favorable for the development of FHB. Disease incidence in the untreated control plots averaged $52 \%$ and severity averaged $10 \%$ (Table 2). Incidence and severity were significantly reduced by single applications of benomyl or tebuconazole $(P=0.05)$. Plots receiving two sprays of benomyl or tebuconazole at Feekes 10.5 and Feekes 11.0 (14 days later) had less FHB than plots receiving one spray at Feekes 10.5 (Table 2), but in no instance were these differences statistically significant $(P \leq 0.20)$. Mancozeb and iprodione did not significantly $(P=0.05)$ reduce $\mathrm{FHB}$, VSK, or DON concentrations in this experiment (Table 2). Benomyl, mancozeb, and tebuconazole significantly reduced $(P=0.05)$ the index of leaf diseases compared to the untreated control (Table 2). The range of differences was 4.7 to 6.4 , suggesting that leaf disease expression was limited to lower and middle leaves (12), and flag leaf damage was not extensive. Grain yields from benomyl-, mancozeb-, and tebuconazole-treated plots were significantly $(P=0.05)$ higher than the yield from plots receiving no fungicide (Table 2). Post-harvest grain quality parameters reflected treatment effects, including increased TW and TKW and reduced \% VSK and DON concentration (Table 2). The relationship between the assessment variables used in this study was examined. A correlation matrix for the variables examined is presented in Table 3 . All correlation coefficients were significant at $P<0.01$.

Reduced-rate and single-application studies on a susceptible cultivar: Crookston. In reduced-rate studies conducted at the NWES in Crookston, the incidence of FHB in unsprayed plots of susceptible cv. Norm averaged $81 \%$ and severity averaged $28 \%$ (Table 4). In a combined ANOVA, there were no significant $(P=0.05)$ treat-

Table 8. Disease assessments and grain quality parameters from the Fusarium head blight (FHB)-susceptible, 6-row malting barley cv. Stander at the Northwest Experiment Station in Crookston, MN in 1995

\begin{tabular}{|c|c|c|c|c|c|c|}
\hline Treatment, a.i./ha ${ }^{a}$ & FHB I $(\%)^{b}$ & FHB S $(\%)^{b}$ & Yield (kg/ha) & Plump (\%) & KD index ${ }^{d}$ & DON $(\mu \mathrm{g} / \mathrm{g})^{\mathrm{e}}$ \\
\hline Benomyl, $560 \mathrm{~g}$ & 91 & 12 & 4,289 & 85.7 & 3.8 & 1.8 \\
\hline Benomyl, $280 \mathrm{~g}+$ Mancozeb, $840 \mathrm{~g}$ & 89 & 11 & 4,728 & 86.6 & 3.9 & 2.4 \\
\hline Benomyl, $280 \mathrm{~g}$ +Propiconazole, $126 \mathrm{~g}$ & 87 & 12 & 4,741 & 87.6 & 3.8 & 2.3 \\
\hline Difenoconazole, $122 \mathrm{~g}$ & 88 & 14 & 4,413 & 86.5 & 3.7 & 4.1 \\
\hline Fenbuconazole, $210 \mathrm{~g}$ & 86 & 14 & 4,940 & 87.2 & 3.8 & 3.6 \\
\hline Fludioxonil, $560 \mathrm{~g}$ & 81 & 8 & 4,699 & 88.0 & 3.8 & 1.6 \\
\hline Mancozeb, $1680 \mathrm{~g}$ & 90 & 15 & 4,177 & 84.8 & 4.0 & 3.6 \\
\hline Propiconazole, $126 \mathrm{~g}$ & 90 & 17 & 4,492 & 85.3 & 3.8 & 3.6 \\
\hline Tebuconazole, $126 \mathrm{~g}$ & 88 & 16 & 4,475 & 86.7 & 3.7 & 2.5 \\
\hline No fungicide & 90 & 16 & 4,253 & 83.8 & 4.0 & 3.4 \\
\hline $\operatorname{LSD}(P=0.05)$ & 5 & 5 & 557 & 2.3 & ns & 0.9 \\
\hline
\end{tabular}

a Treatments applied at Feekes 10.5.1. Latron CS-7 at $120 \mathrm{ml} / \mathrm{ha}$ was added to benomyl and mancozeb treatments as per the manufacturer's recommendations. LSD = least significant difference; $n s=$ not significant.

b Fusarium head blight incidence (I) and severity (S) based on assessment of 1,080 heads.

c Percentage of $250 \mathrm{~g}$ of kernels that failed to pass a 2.38-by-19.05-mm slotted screen (4).

d Kernel discoloration indexed on a 1-to-5 scale according to the methods of Miles et al. (22).

e Deoxynivalenol concentration. 
ment-year interactions and data were combined for presentation. A single-application tank mix of benomyl (280 $\mathrm{g}$ a.i./ha) plus mancozeb (840 g a.i./ha) reduced FHB incidence by $16 \%$ and FHB severity by $12 \%$. Applications of benomyl $(280 \mathrm{~g}$ a.i./ha) plus tebuconazole (126 g a.i./ha) reduced FHB incidence by $15 \%$ and reduced FHB severity by $11 \%$ (Table 4 ). Fludioxonil (560 g a.i./ha) and mixtures of carbendazim (133 $\mathrm{g}$ a.i./ha) plus tebuconazole (167 $\mathrm{g}$ a.i./ha) also resulted in significant $(P=0.05)$ reductions in FHB incidence and severity (Table 4$)$. Difenoconazole at $245 \mathrm{~g}$ a.i./ha reduced FHB incidence and severity $(P=0.05)$. Difenoconazole at $122 \mathrm{~g}$ a.i./ha, fenbuconazole (210 g or $420 \mathrm{~g}$ a.i./ha), and mancozeb $(1680 \mathrm{~g}$ a.i. /ha) resulted in mean scores lower than, but not statistically $(P=0.05)$ different from, the untreated control (Table 4). Leaf diseases, primarily tan spot and Stagonospora leaf and glume blotch, were present in these studies and Norm developed levels of these diseases that caused damage to the flag leaf and the f-1 leaf (Hosford scale readings >6.0). All fungicide treatments reduced $(P=0.05)$ the leaf disease index in these experiments (Table 4).

Grain yield and post-harvest grain quality parameters reflected FHB development in these studies. Effective treatments increased yield and TW while reducing the $\%$ VSK and DON concentration in grain (Table 4).

Reduced-rate and single-application studies on a susceptible cultivar: Morris. In mist-irrigated experiments conducted in 1995 and 1996 at the WCES in Morris, the incidence of FHB in unsprayed plots of susceptible cv. Norm averaged $85 \%$ and FHB severity averaged $29 \%$ (Table 5). In a combined ANOVA, there were no significant $(P=0.05)$ treatment-year interactions and data were combined for presentation. A single application of benomyl $(560 \mathrm{~g}$ a.i./ha) reduced incidence by $31 \%$ and reduced severity by $17 \%$ (Table 5 ). A tank mix of benomyl (280 $\mathrm{g}$ a.i./ha) plus tebuconazole (126 g a.i./ha) reduced incidence by $25 \%$ and reduced severity by $14 \%$. Mixtures of benomyl (280 g a.i./ha) plus mancozeb (840 g a.i./ha) or benomyl (280 $\mathrm{g}$ a.i./ha) plus propiconazole (126 g a.i./ha) significantly $(P=0.05)$ reduced FHB incidence and severity (Table 5 ). Fludioxonil $(560 \mathrm{~g}$ a.i./ha), tebuconazole (126 g a.i./ha), or propiconazole (126 g a.i./ha) also reduced FHB incidence and severity (Table 5). None of these treatments were significantly different $(P=0.05)$ from each other. Azoxystrobin (224 or $448 \mathrm{~g}$ a.i./ha) reduced FHB incidence and severity compared to the unsprayed control but the lower rate resulted in significantly $(P=$ $0.05)$ more FHB than other treatments (Table 5). Leaf disease indices were significantly $(P=0.05)$ reduced by all treatments except benomyl alone. Mean scores did not exceed 6.0 on the Hosford scale (12), and damage was observed only on lower and middle leaves.

Yield and post-harvest grain quality parameters reflected FHB development. Effective treatments increased yield and TW while reducing the \% VSK and the DON concentration in grain (Table 5). Azoxystrobin at 224 or $448 \mathrm{~g}$ a.i./ha reduced FHB and leaf diseases and increased yield, but differences in TW, \% VSK, and DON were not significant $(P=0.05)$ except at the higher rate (Table 5).

Reduced-rate and single-application studies on an MS cultivar. In three of four trials, single applications of azoxystobin (224 g a.i./ha), propiconazole (126 g a.i./ha), or tebuconazole (126 g a.i./ha) applied to MS cv. 2375 at Feekes 10.5.1 significantly $(P=0.05)$ reduced the incidence and severity of FHB compared to the untreated control. In one trial (wheat planted after fallow), the incidence and severity of FHB in unsprayed plots averaged less than 2 and $1 \%$, respectively; the $\%$ VSK averaged 1.3 and the mean DON concentration was $0.9 \mu \mathrm{g} / \mathrm{g}$. Further results from this trial are not presented. In the remaining three trials, ANOVA revealed no significant $(P=0.05)$ treatment-site (location) interactions and data were combined for presentation (Table 6). Propiconazole and tebuconazole treatment significantly $(P=0.05)$ reduced FHB incidence and severity compared to plots treated with azoxystrobin (Table 6). All fungicide treatments significantly $(P=$ 0.05 ) reduced leaf diseases (primarily Stagonospora leaf and glume blotch) compared to the untreated control (Table 6). Yield and TW were significantly $(P=$ $0.05)$ increased and the \% VSK and DON concentration were significantly $(P=0.05)$ reduced with fungicide treatment (Table 6).

Cultivar-fungicide interaction study. FHB incidence among the unsprayed treatments of seven spring wheat cultivars evaluated in 1995 ranged from 86\% (cv. Norm) to $62 \%$ (cv. Marshall), and significant differences $(P=0.05)$ were observed between Norm and other less susceptible cultivars (Table 7). FHB severity ranged from $40 \%$ (Norm) to $10 \%$ (Marshall). Treatment with a mixture of benomyl (280 g a.i./ha) plus propiconazole (126 g a.i./ha) at Feekes 10.5.1 (15 to $25 \%$ anthesis) reduced FHB incidence in all cultivars and differences were significant $(P=0.05)$ for cvs. Butte 86, Norm, Sharp, and Verde (Table 7). Fungicide treatment significantly reduced FHB severity in all cultivars. Treatment differences were highly significant $(P=0.01)$ between sprayed and unsprayed plots of 2375, Butte 86, Norm, and Sharp. Treatment differences were significant $(P=0.05)$ between sprayed and unsprayed plots of Grandin, Marshall, and Verde (Table 7). Leaf disease ratings were variable among cultivars and significantly different between sprayed and unsprayed treatments of all cultivars except Norm (Table 7). Yield and post-harvest grain quality parameters varied between cultivars and between sprayed and unsprayed treatments of the same cultivar, but reductions in DON as a result of fungicide treatment were only significant $(P=0.05)$ in the case of Grandin (Table 7).

Barley studies. In 1995, the incidence of FHB on unsprayed treatments of Stander barley at Crookston averaged 90\% and FHB severity averaged $16 \%$ (Table 8 ). Fludioxonil (560 g a.i./ha) significantly $(P$ $=0.05$ ) reduced FHB incidence to $81 \%$ and severity to $8 \%$. FHB incidence was not significantly reduced with other treatments $(P=0.05)$. A tank mix of benomyl $(280 \mathrm{~g}$ a.i./ha) plus mancozeb (840 g a.i./ha) significantly $(P=0.05)$ reduced FHB severity to $11 \%$. Fenbuconazole (210 g a.i./ha) application resulted in significantly $(P=$ $0.05)$ higher yields. Other treatments did not significantly $(P=0.05)$ affect yield. Certain of the fungicide treatments increased plump kernels $(P=0.05)$ compared to the untreated control (Table 8$)$ and benomyl (560 $\mathrm{g}$ a.i./ha), benomyl (280 g a.i./ha) plus mancozeb (840 g a.i./ha), benomyl (280 $\mathrm{g}$ a.i./ha) plus propiconazole (126 g a.i./ha), fludioxonil (560 g a.i./ha), and tebuconazole (126 $\mathrm{g}$ a.i./ha) reduced DON concentrations $(P=0.05)$. KD, a disease primarily associated with late-season colonization of Bipolaris sorokiniana (Sacc.) Shoemaker (22), was not affected by any of the fungicide sprays in this test (Table 8).

In 1996, dry weather 3 weeks before and 4 weeks after heading affected FHB development in the barley fungicide experiment at Crookston. The incidence of FHB in unsprayed plots averaged $10 \%$ and FHB severity averaged $2 \%$. FHB incidence and severity averaged 7 and $1 \%$ respectively, across all fungicide treatments. The lowest treatments were benomyl (560 g a.i./ha), benomyl (280 g a.i./ha) plus mancozeb (840 g a.i./ha), benomyl (560 g a.i./ha) plus propiconazole (126 g a.i./ha), and fludioxonil (560 g a.i./ha) with 5 and $1 \%$ FHB incidence and severity, respectively. These differences were not significantly different $(P=0.05)$ from the untreated control. Yield averaged 6,539 kg/ha (range: 6,417 to $6,753 \mathrm{~kg} / \mathrm{ha}$ ). Differences in yield between treatments were not statistically significant $(P=0.05)$. Plump kernels ranged from 86.8 to $91.5 \%$ across treatments; differences were not significant $(P$ $=0.05)$. KD scores ranged from a high of 3.7 (no fungicide) to a low of 2.5 for the benomyl (280 g a.i./ha) plus mancozeb (840 g a.i./ha) treatment; differences were significant $(P=0.05)$. Concentrations of DON averaged less than $1 \mu \mathrm{g} / \mathrm{g}$ and differences between treatments were not significant $(P=0.05)$. Due to the low levels of disease in the 1996 experiment, results were not combined with results obtained in 1995. 
Fungicidal activity in vitro. Mycelial growth inhibition increased with increasing concentrations of (i) benomyl, (ii) fludioxonil, (iii) mancozeb, and (iv) tebuconazole. The mean $\mathrm{ED}_{50}$ values for isolates SP4, M6, and CD1 tested against these four fungicides were $9.9 \times 10^{-1}, 7.4 \times 10^{-3}$, 5.8, and $3.5 \times 10^{-1} \mathrm{mg}$ a.i./liter, respectively. The mean $\mathrm{ED}_{90}$ values were 7.3, 1.1 $\times 10^{-1}, 67.7$, and $7.3 \mathrm{mg}$ a.i./liter, respectively. The radial growth inhibition of isolates SP4, M6, and CD1 is illustrated in Figure 1. The mean radial growth rate for these isolates on unamended PDA (zero concentration) was $0.45 \mathrm{~mm} / \mathrm{h}$ and isolate differences were significant $(P=0.01)$. When comparing $\mathrm{ED}_{50}$ values for growth inhibition of $F$. graminearum, almost 10fold differences were observed between fungicides: fludioxonil > tebuconazole > benomyl > mancozeb. When comparing $\mathrm{ED}_{90}$ values, growth rate inhibition response changed somewhat with fludioxonil $>$ benomyl = tebuconazole $>$ mancozeb (Fig. 1). The collection of 50 isolates of $F$. graminearum ( $G$. zeae) obtained from R. D. Wilcoxson (37) grew at a rate of 0.46 $\mathrm{mm} / \mathrm{h}$ on unamended PDA plates but failed to grow on plates amended with benomyl, fludioxonil, or tebuconazole at $10 \mathrm{mg} / \mathrm{liter}$. This suggests an absence of background levels of resistance in $F$. graminearum populations to these fungicides because these isolates had been collected prior to 1990 and prior to any widespread commercial use of these fungicides on wheat or barley in Minnesota. Of 50 isolates, 10 failed to grow on plates amended with mancozeb at $10 \mathrm{mg} / \mathrm{liter}$. The remaining 40 isolates averaged $9.5 \mathrm{~mm}$ of growth $(72 \%$ inhibition) at this dose.

Wheat seed exposed to applications of benomyl at 560 or $1,120 \mathrm{~g}$ a.i./ha (500 seed each assayed from treatments in experiments at WCES, Morris in 1994, 1995, and 1996) failed to produce colonies of $F$. graminearum on PDA plates amended with benomyl at $10 \mathrm{mg} / \mathrm{liter}$. The mean radial growth of 60 seed (20 per experimental run) individually plated on unamended PDA (unamended control) was $31.4 \mathrm{~mm}$. Background levels of resistance or insensitivity to benomyl were not observed in this study, nor did resistant strains develop following three consecutive years of benomyl applications to the field trial sites.

\section{DISCUSSION}

Heading treatments of fungicides significantly reduced FHB and other key parameters of the disease that contribute to yield and quality reductions. Among those tested, the methyl benzimidizole carbamates (MBC) fungicides, which include benomyl, and triazole fungicides (DMI or SI), which include tebuconazole, exhibit the most consistent ability to reduce FHB in the field and to reduce the incidence of Fusarium spp.-damaged kernels (\% VSK) and DON concentration in harvested grain. In all studies, these factors were correlated with one another. Benlate (benomyl) is

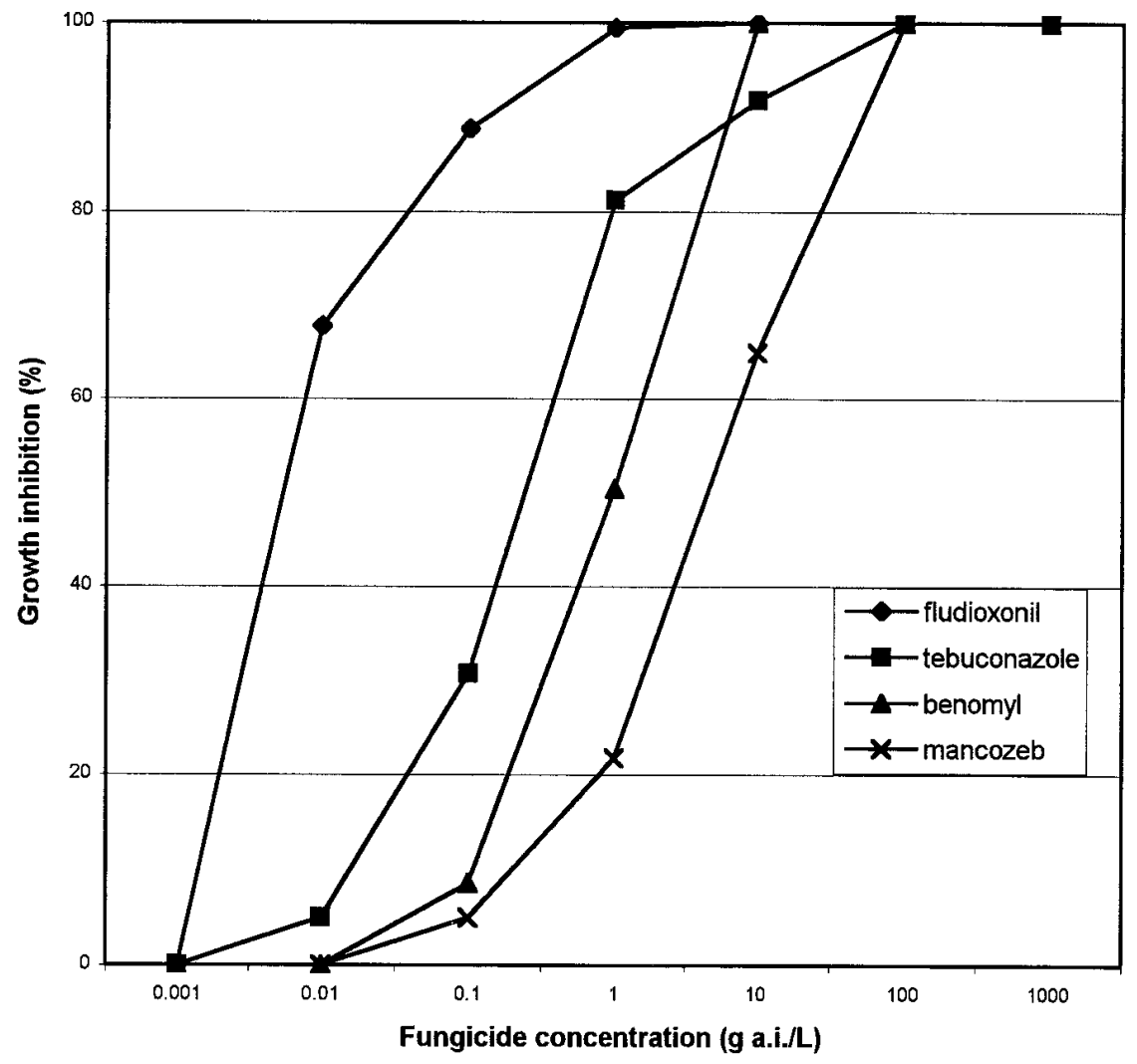

Fig. 1. Growth rate inhibition of Fusarium graminearum isolates SP4, M6, and CD1 at selected fungicide concentrations. currently registered in the United States for heading applications on wheat (including durum) but is not registered on barley. The MBC fungicides, including benomyl, carbendazim, and thiophanate methyl, have been found to reduce the severity of FHB epidemics in South America, East Asia, and certain areas of Europe $(11,24,31)$. In these areas, $F$. graminearum ( $G$. zeae) predominates among the mixtures of Fusarium spp. present in regional populations.

Some triazole fungicides, including tebuconazole, were as effective as benomyl in reducing FHB severity. In several experiments, propiconazole was not different from tebuconazole in reducing the incidence and severity of FHB. Difenoconazole was less effective in reducing FHB severity than tebuconazole or propiconazole and fenbuconazole was ineffective. Suppression of FHB with triazoles is highly dependant on application rate and frequency $(20,31)$. In inoculated-misted nurseries in Minnesota, FHB can be completely controlled with six to eight applications of $2 \times$ rates of tebuconazole or tebuconazole plus carbendazim (J. V. Wiersma, Minnesota Agricultural Experiment Station, Crookston; personal communication). Spray regimes such as these would not be economical for commercial producers. At lower rates (126 to $380 \mathrm{~g}$ a.i./ha), and less frequent applications, varying levels of disease suppression can be achieved. Triazoles, including tebuconazole and propiconazole, were very effective in reducing the severity of foliar diseases, including Stagonospora leaf and glume blotch (S. nodorum) and Stagonospora leaf blotch (S. avenae f. sp. triticea). Benomyl was less effective.

Application timing is an important factor in effectively suppressing FHB with fungicides. In Europe, Mauler-Machnik and Zahn (20) summarized the results of over 30 trials conducted in France from 1986 through 1993. Applications of tebuconazole (Folicur 250EC at 1.0 liters/ha) at anthesis reduced FHB incidence from 41 to $16 \%$, reduced infected kernels from 28 to $12 \%$, and increased yield by $34 \%$ compared to unsprayed controls. Suty and Mauler-Machnik (31) also reported extensively on the results of tebuconazole applications. Results in this study agree with their results. Applications of fungicides at or just after beginning anthesis are beneficial in reducing FHB, reducing the \% VSK in harvested grain, and reducing concentrations of DON.

Certain nonsystemic fungicides, including fludioxonil (a Class $\mathrm{E}$ oncogen) were effective in reducing FHB and DON contamination of wheat and barley. Mancozeb significantly reduced FHB assessments in certain experiments; however, the performance of mancozeb was not as good nor as consistent as other treatments. Azoxystrobin was intermediate and iprodione 
and triphenyl hydroxide were ineffective compared to benomyl or tebuconazole. Fludioxonil was slightly more effective and benomyl was slightly less effective on barley compared to wheat.

The variability in assessment data associated with measures of FHB in field studies complicates other research efforts directed towards evaluating management practices. Reductions in detached head weight, TW, and yield are all indirect indicators of disease. Although correlated with disease severity, these measures can be confounded with other spray-treatment effects and may not be the best measures for determining spray efficacy. For example, in trials involving cv. 2375, it was not possible to determine if the yield and TW effects of triazole sprays were the result of reducing FHB or the result of reducing fungal leaf diseases. It may result from a combination of both factors that is difficult to partition. However, reductions in \% VSK and reductions in DON can be directly attributed to reductions in FHB. The high levels of naturally occurring FHB, combined with selecting cv. Norm (resistant to many of the foliar diseases present), did facilitate the ability to differentiate chemical treatment responses in relationship to FHB in this study.

Wheat grains are variably invaded by $F$. graminearum $(6,7,16,33,37,38)$. Recovery of viable propagules of the fungus (expressed as the number of infected kernels) gives little indication of disease severity. Bekele et al. (6) reported that visual ratings of FHB were correlated with kernel infection, but that visual score and mummified (tombstone) kernels were not always correlated. Boyacioglu et al. (7), however, reported up to $19 \%$ infected kernels from spring wheat with no symptoms of FHB. In this study, \% VSK was found to be an accurate and rapid measure of Fusarium spp.-damaged kernels (FDK) that correlated with DON. A weight-percentage count of FDK is used by grain inspectors to determine the grade of grain offered for sale (4) and is a factor in the financial loss associated with FHB epidemics (21).

The best measures of FHB include visual assessments of disease incidence and disease severity (evaluating a constant, but reasonably large number of heads). These parameters, combined with some measure of FDK (such as \% VSK), and quantifying DON concentrations in grain, provide consistent assessment of the disease and reflect treatment differences. As reported here, the best indicator variables were percent FHB incidence, percent FHB severity, $\%$ VSK, and DON. The first three of these variables are relatively inexpensive to obtain, whereas the latter costs approximately $\$ 20$ to 25 per analysis. Additional factors to consider when screening fungicides against FHB include increasing replication; avoiding wheat as a previous crop (corn is more useful in producing suitable, uniform inoculum); avoiding artificial inoculation; and planting FHB-susceptible cultivars with high levels of resistance to other, potentially confounding diseases.

The severity scale developed by Chelkowski (8) and modified here combines rapidity with precision. It is faster than individually counting symptomatic spikelets (38). It is not as difficult as the Horsfall-Barrett scale or the visual scale for FHB proposed by Stack and McMullen (30). These scales contain too many levels of disease for rapid evaluation of large numbers of heads. Because of the inherent variability in assessing FHB, assessments of a greater number of heads improves the accuracy of disease severity estimates compared to assessing fewer heads more carefully. The addition of a severity class 6 (93.75\% disease) to Chelkowski's original scale (8) allows more precision and, when working with susceptible cultivars, more accurate mean scores and better mean separation can be achieved.

In vitro analysis suggests that isolates of $F$. graminearum are sensitive to benomyl or tebuconazole at a rate of $10 \mathrm{mg}$ of the active ingredient per kilogram. Sensitivity to mancozeb was one order of magnitude less and that of fludioxonil was one order of magnitude greater than benomyl or tebuconazole (Fig. 1). Growth inhibitions at these concentrations suggest that the relatively poor performance of these fungicides in the field tests is not related to their inactivity against the causal organism. It also supports observations about the effectiveness of ethylene bisdithiocarbamates (EBDCs), triazoles, and the MBC fungicides (including thiabendazole) as seed treatments and their ability to control the seedling blight phase of this disease (16).

Systemic fungicides may result in less FHB because they are absorbed into tissues and are less subject to removal by moisture. Effective triazoles (tebuconazole, propiconazole) may also have some postinfection activity $(20,31)$. The architecture of wheat or barley heads in relationship to spray patterns and spray deposition would suggest that a low percentage of fungicide is delivered to the infection court. However, in the experiment summarized in Table 2, the percent reduction in key FHB assessment parameters was not related to dose. In this trial, two applications (14 days apart) were not statistically better than a single application. Suty and MaulerMachnik (31) suggest that split applications of tebuconazole are beneficial when the time between sprays is reduced to 7 days. Additional studies investigating application timing, fungicide formulation, and an analysis of the benefits of spray additives are needed.

MBC insensitivity (resistance) may become a concern if benomyl sprays are widely adopted in the United States. In Europe, triazole fungicides are more effec- tive than the MBC types, including carbendazim $(20,31)$ and benomyl $(20,31)$. However, the etiological components of FHB in Western Europe differ from those in the United States in that regions of intensive wheat cultivation, where production economics support chemical control options, are not the geographical regions where $F$. graminearum (G. zeae) predominates. Also, insensitive populations of Gerlachia nivalis, considered a component of the head blight complex in Europe $(25,26)$, may be responsible for the reduced efficacy of benomyl especially in Scandinavia (Sweden, Norway, and Finland) and other regions where the snow mold fungus is well adapted.

Valid concern exists regarding treatment economics for control of FHB using fungicides. Establishing a cost-benefit relationship to analyze net return from treating wheat or barley is difficult. At all rates tested, good control was not achieved and left a harvested product containing varying levels of FDKs and DON, whose value in the marketplace is highly variable and difficult to estimate. The gross return on the absolute yield difference between sprayed and unsprayed plots exceeds an estimated program cost for applications of benomyl plus mancozeb, tebuconazole, or propiconazole on highly susceptible spring wheat cultivars. However, these cultivars were immediately abandoned by producers after the epidemics of 1993 and 1994 $(18,21)$. In spring wheats that are MS to FHB, gross return from a single (low-rate) application of fungicide appears to be more a function of control of Septoria diseases and tan spot than benefits resulting from direct reductions in FHB. This shifts the treatment efficacy in favor of the triazoles and away from benomyl. In barley, malt buyers are currently maintaining a nearzero tolerance for DON. None of the tested sprays were found to eliminate this mycotoxin in barley.

Food safety issues continue to impact chemical management options for controlling FHB. High rates and multiple applications affect control costs and also heighten residue concerns in products marketed from wheat and barley. At the present time, new fungicides that are highly effective against Fusarium spp., cost beneficial, and that result in undetectable residues in wheat or barley do not appear to be in the near stages of development. For this reason, any short-term role that fungicides will play in managing severe outbreaks of FHB in the United States appears minimal.

\section{LITERATURE CITED}

1. Almeras, R. R., Wilkins, D. E., Burnside, O. C., and Mulla, D. J. 1998. Agricultural technology and adoption of conservation tillage practices. Pages 99-158 in: Advances in Soil and Water Conservation. F. J. Pierce and W. W. Frye, eds. Ann Arbor Press, Chelsea, MI.

2. Anderson, P. M., Oelke, E. A., and Simmons, S. R. 1985. Growth and development guide 
for spring barley. Agric. Ext. Serv. Univ. Minn. AG-FO-2548.

3. Anonymous. 1986. Guidelines for Carcinogens Risk Assessment. Federal Register 51:33992-34003. Sept. 24, 1986. U. S. Gov. Print. Off. Washington, DC.

4. Anonymous. 1991. Inspecting Grain, Practical Procedures for Grain Handlers. U. S. Dep. Agric. Fed. Grain Inspection Serv. U. S. Gov. Print. Off. Washington, DC.

5. Anonymous. 1994. Varietal Trials of Selected Farm Crops. MR-5615-F. Minn. Agric. Exp. Stn.

6. Bekele, G. T., Wilcoxson, R. D., Sugunda, T., Busch, R. H., and Warnes, D. D. 1994. Comparison of methods for estimating head blight reactions of spring wheat cultivars infected with Fusarium graminearum. Int. J. Trop. Plant Dis. 12:89-100.

7. Boyacioglu, D., Hettiarachchy, N. S., and Stack, R. W. 1992. Effect of three systemic fungicides on deoxynivalenol (vomitoxin) production by Fusarium graminearum in wheat. Can. J. Plant Sci. 72:93-101.

8. Chelkowski, J. 1989. Formation of mycotoxins produced by Fusaria in heads of wheat, triticale and rye. Pages 63-84 in: Fusarium: Mycotoxins, Taxonomy, and Pathogenicity. J. Chelkowski, ed. Elsevier Press, Amsterdam.

9. Dill-Macky, R., and Jones, R. K. 2000. The effect of previous crop residues and tillage on Fusarium head blight of wheat. Plant Dis. 84:71-76.

10. Fehrman, H., and Ahrens, W. 1984. Attack of wheat by Septoria nodorum and Fusarium ear scab. II. Spraying curatively acting fungicides. J. Plant Dis. Prot. 91:113-121.

11. Galich, M. T. 1989. Importancia y distribucion de la fusariosis del trigo en Argentina. Pages 7-26 in: Taller sobre la fusariosis de la espiga en America del Sur. M. M. Kohli, ed. Mexico, D.F. CIMMYT.

12. Hosford, R. M., Jr. 1982. Tan spot. Pages 1-24 in: Tan Spot of Wheat and Related Diseases Workshop. R. M. Hosford, Jr., ed. N. D. State Univ. Fargo.

13. Hutcheon, J. A., and Jordan, V. W. L. 1992. Fungicide timing and performance for Fusarium control in wheat. Pages 633-638 in: Brighton Crop Prot. Conf.-Pests Dis.

14. Jacobsen, B. J. 1977. Effect of fungicides on Septoria leaf and glume blotch, Fusarium scab, grain yield, and test weight of winter wheat. Phytopathology 67:1412-1414.
15. Jones, R. K. 1994. Managing Fusarium head scab for 1995. Plant Pest Newsl. PPST 17:100-102. Minn. Ext. Serv. Univ. Minn. St. Paul.

16. Jones, R. K. 1999. Seedling blight development and control in spring wheat damaged by Fusarium graminearum group 2. Plant Dis. 83:1013-1018.

17. Jones, R. K., Belmar, S. B., and Jeger, M J. 1987. Evaluation of benomyl and propiconazole for controlling rice sheath blight. Plant Dis. 71:222-225.

18. Jones, R. K., and Mirocha, C. J. 1999. Quality parameters in small grains from Minnesota affected by Fusarium head blight. Plant Dis. 83:506-511.

19. Martin, R. A., and Johnston, H. W. 1982. Effects and control of Fusarium diseases of cereal grains in the Atlantic Provinces. Can. J. Plant Pathol. 4:210-216.

20. Mauler-Machnik, A., and Zahn, K. 1994. Ear fusariosis in wheat-new findings on their epidemiology and control with Folicur (tebuconazole). Pflanzenschutz-Nahr. Bayer (Ger. Ed.) 47:129-153.

21. McMullen, M. P., Jones, R. K., and Gallenberg, D. J. 1997. Scab of wheat and barley-A re-emerging disease of devastating impact. Plant Dis. 81:1340-1348.

22. Miles, M. R., Wilcoxson, R. D., Rasmusson, D. C., Wiersma, J., and Warnes, D. 1987. Influence of genotype and environment on kernel discoloration of Midwestern malting barley. Plant Dis. 71:500-504.

23. Milus, E. A., and Parsons, C. E. 1994. Evaluation of foliar fungicides for controlling Fusarium head blight of wheat. Plant Dis. 78:697-699.

24. Neto, N., and Giordani, N. A. 1989. Control quimico de la fusariosis en trigo. Pages 109118 in: Taller sobre la fusariosis de la espiga en America del Sur. M. M. Kohli, ed. Mexico, D.F. CIMMYT.

25. Olvang, H. 1984. Benomyl resistance in Gerlachia nivalis. I. Survey of diseased plants in the field. Z. Pflanzenkrankh. Pflanzenschutz. 91:294-300.

26. Olvang, H. 1987. Benomyl resistance in Gerlachia nivalis. III. Alternatives to the benzimidizole-fungicides for foliar application. Z. Pflazenkrankh. Pflanzenschutz. 94:242249.

27. Saari, E. E., and Prescott, J. M. 1975. A scale for appraising the foliar intensity of wheat diseases. Plant Dis. Rep. 59:377-380.

28. Schroeder, H. W., and Christensen, J. J. 1963 Factors affecting resistance of wheat to scab caused by Gibberella zeae. Phytopathology 53:831-838.

29. Simmons, S. R., Oelke, E. A., and Anderson, P. M. 1985. Growth and development guide for spring wheat. Agric. Ext. Serv. Univ. Minn. AG-FO-2547.

30. Stack, R. W., and McMullen, M. P. 1995. A visual scale to estimate severity of Fusarium head blight in wheat. PP-1095. N. D. State Univ. Ext. Serv. Fargo.

31. Suty, A., and Mauler-Machnik, A., 1996. Fusarium head blight on wheat-new findings on the epidemiology and control of Gibberella zeae the teleomorph of Fusarium graminearum with Folicur. PflanzenschutzNahr. Bayer (Ger. Ed.) 49:55-70.

32. Ueda, S., and Yoshizawa, T. 1988. Effect of thiophanate methyl on the incidence of scab and the mycotoxin contamination in wheat and barley. Ann. Phytopathol. Soc. Jpn. 54:476-482.

33. Wainwright, A., Jeitner, J., and CazinBourguignon, P. 1992. Reduction in the wheat ear disease complex with tebuconazole sprays. Pages 621-626 in: Brighton Crop Prot. Conf.-Pests Dis.

34. Wiersma, J. V., Peters, E. L., Hanson, M. L., Bouvette, R. J., and Busch, R. H. 1996. Fusarium head blight in hard red spring wheat: cultivar responses to natural epidemics. Agron. J. 88:223-230.

35. Wilcoxson, R. D. 1996. Fungicides for control of Fusarium head blight-A review. Minn. Agric. Exp. Stn. Paper no. 22,507. St. Paul.

36. Wilcoxson, R. D., Busch, R. H., and Ozmon, E. A. 1992. Fusarium head blight resistance in spring wheat cultivars. Plant Dis. 76:658-661.

37. Wilcoxson, R. D., Kommedahl, T., Ozmon, E. A., and Windels, C. E. 1988. Occurrence of Fusarium species in scabby wheat from Minnesota and their pathogenicity to wheat Phytopathology 78:586-589.

38. Wilcoxson, R. D., Ozmon, E. A., and Pierce, A. R. 1989. Effect of necrotic spikelets and location of plots on infection of wheat cultivars by Fusarium graminearum. Int. J. Trop. Plant Dis. 7:55-60.

39. Woodward, R. P., and Wilcoxson, R. D. 1996. Pathogenic variation of Fusarium graminearum. Recent Res. Dev. Plant Pathol. 1:73-81. 